\title{
Concentração de fluoreto na vegetação próxima do pólo de fertilizantes de Cubatão, São Paulo, Brasil
}

\author{
Fluoride concentration in the adjacent vegetation \\ next to fertilizer industries of Cubatão, São Paulo State, Brazil
}

Priscilla Torres Tagawa ${ }^{1}$

Durval Libutti M oruzzi ${ }^{2}$

JaimeA parecido Cury ${ }^{1}$
${ }^{1}$ FaculdadedeOdontologia dePiracicaba, Universidade Estadual deCampinas. Av. Limeira 901, Caixa Postal 52. 13414-903 Piracicaba SP.

prittagawa@hotmail.com ${ }^{2}$ PrefeituraM unicipal de Santos.
Abstract Theaim of this study wasto evaluateif fluoride pollution found in 1996 in Cubatão, SP, Brazil, using the vegetation as biomarker, changed in the last ten years. Leaves of Terminalia cattappa located at the industrial area around the industries of fertilizers and in the urban area of the city were collected in 1996 and 2006. They were dried, powdered and fluoride water soluble extracted was analyzes with specific electrode. The fluoride concentration in the leaves around fertilizer industries was 12 times greater than that found in the urban area, either in 1996 or 2006, respectively. The data suggest that in the last ten years the environmental pollution by fluoride in Cubatão has not improved.

Key words Fluoride, Pollution, Fertilizer industries
Resumo 0 objetivo destetrabalho foi avaliar sea poluição por fluoreto observada em 1996 em Cubatão (SP), utilizando a vegetação como biomarcador, teve alterações nos últimos dez anos. FoIhas de Terminalia cattappa (chapéu do sol), Iocalizadas na região das indústrias de fertilizantes e na área urbana municipal, foram coletadas em 1996 e2006. Asfolhas foram desidratadas, pulve rizadas efluoreto extraído com água foi analisado com el etrodo específico. A concentração de fluoreto encontrada nas folhas das árvores localizadas ao redor do pólo de fertilizantes foi doze vezes maior do que a naquelas da área urbana, tanto em 1996 como 2006. Sugere-se que a poluição ambiental por fluoreto em Cubatão não apresentou melhoria em dez anos.

Palavras-chave Flúor, Poluição, Indústria defertilizantes 
Introdução

Fluoreto é um poluente ambiental industrial ${ }^{1} \mathrm{e}$ entre as fontes poluidoras estão as indústrias de alumínio, cerâmicas, vidros, aço, ácido fosfórico e tijolos, com destaque para a de fertilizantes ${ }^{2}$. Esta poluição podeatingir diretamente não só os trabalhadores destas indústrias ${ }^{3}$, como pode se propagar à distância, provocando desequilíbrio no ecossistema 4 .

O município deCubatão (SP) possui um grande número de indústrias de fertilizantes e 0 impacto da poluiçãa por fluoreto na vegetação da Serra do $M$ ar tem sido relatado desde 19945 . As plantas podem ser usadas para o biomonitoramento da qualidade do $\mathrm{ar}^{2}$ e, através das folhas, é possível medir o acúmulo e a concentração do fluoreto no tecido foliar. Assim, em folhas de Terminalia cattappa, popularmente conhecida como chapéu do sol, localizadas próximas às fábricas de fertilizantes de Cubatão, foi encontrada em 1996 uma alta concentração de fluoreto ${ }^{6}$.

Deste modo, o objetivo desta comunicação é divulgar dados comparativos de 1996 e 2006 da variação da concentração de fluoreto nas folhas de arvores do município de Cubatão, como biomarcador de mudança da qualidade do ar.

\section{M ateriais emétodos}

\section{Amostragem}

Folhas deárvores da espéciearbórea Terminalia cattappa, nome popular chapéu do sol, localizadas ao redor de oito fábricas de fertilizantes de Cubatão (SP) e em quatro locais da zona urbana fora do complexo industrial deste município, foram colhidas dos mesmos lugares nos anos de 1996 e 2006. Asfolhas foram col hidas no segundo semestre de cada ano, em dias sem chuva e no período matutino. Foram coletadas três folhas do gal ho mais baixo, aproximadamente $1,70 \mathrm{~m}$, na terceira ramificação, sendo uma folha nem muito nova (verde epequena) enem muito velha (seca e queimada), e foram colocadas em sacos de papel. Os sacos foram codificados, sendo 0 estudo cego com relação à analise laboratorial.

\section{Extração e determinação do fluoreto}

As fol has foram picotadas, desidratadas a 900 $C$ por vinte horas, moídas e duplicatas de 5 a 15 $\mathrm{mg}( \pm 0,01 \mathrm{mg})$, foram pesadas em tubos de ensaio plásticos. Um mililitro de água destilada e deionizada foi adicionada aos tubos e após trinta minutos sob agitação a $370 \mathrm{C}$, 0 extrato foi tamponado com 1,0 mL de TISAB II [tampão acetato $1 \mathrm{M}$ pH 5,0, contendo $\mathrm{NaCl} 1 \mathrm{M}$, eCDTA (ácido 1,2 ciclohexanodiaminotetracético a $0,4 \%$ )] 0 fluoreto extraído foi determinado com eletrodo especifico Orion 96-09 e analisador de íons O rion EA-940, previamente calibrados com padrões de íon flúor de 0,05 a $20 \mu \mathrm{g} \mathrm{F} / \mathrm{mL}$. A variabilidade média entre as duplicatas foi menor que $9 \%$. A metodologia de extração quanto à secagem, peso de amostra e temperatura de extração foi previamente padronizada.

\section{Análise estatística}

Os dados foram analisados estatisticamente utilizando o software Bioestat ${ }^{7}$ a $5 \%$ de significância ea diferença de concentração entreos anos de1996 e 2006 para cada região foi estimada pelo teste t pareado.

\section{Resultados}

A Figura 1 mostra os resultados das médias e desvios padrões das concentrações de fluoreto encontradas em 1996 e 2006 nas duas regiões do município de Cubatão. A concentração na vegetação ao redor de oito indústrias de fertilizantes variou de 33,8 a 363,9 e 65,3 a 470,4 $\mu \mathrm{g} \mathrm{F/g} \mathrm{ena}$ zona urbana fora do complexo industrial $(n=4)$

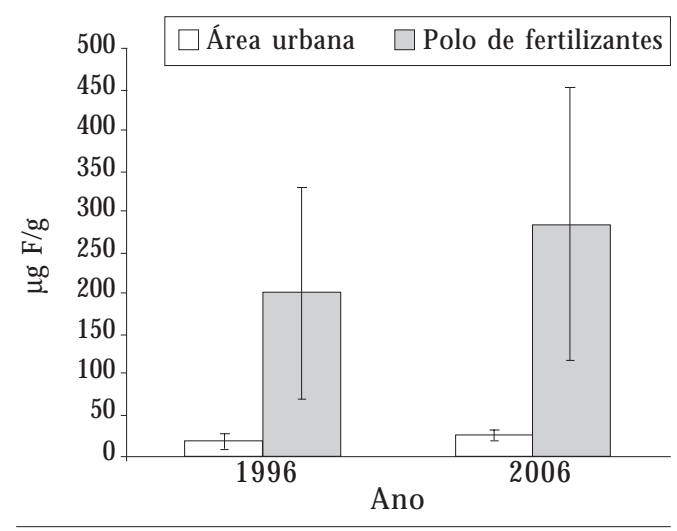

Figura 1. M édias (DP) da concentração de fluoreto ( $\mu \mathrm{g} \mathrm{F/g}$ peso seco de folha) encontradas em $1996 \mathrm{e}$ 2006 nas folhas de T. cattappa localizadas na área urbana ( $n=4)$ e no pólo industrial de fertilizantes ( $n=8)$ de Cubatão, São Paulo, Brasil. 
variou de 10,8 a 33,2 e 16,0 a 30,4 $\mu \mathrm{g} \mathrm{F/g} \mathrm{em} 1996$ e 2006, respectivamente. A diferença de média entre os anos de avaliação para cada região não foi estatisticamente significativa $(p>0,05)$, sendo significante entre regiões $(p<0,05)$.

\section{Discussão}

0 efeito da poluição ambiental por fluoreto pelo complexo industrial de Cubatão na vegetação nativa da Serra do $M$ ar tem sido avaliado em folhas de plantas das espécies Tibouchina pulchra, M iconia pyrifolia e Cecronia glazioui ${ }^{2}$. No presente estudo, utilizamos folhas de Terminalia catappa, popularmente conhecida por chapéu do sol, porque esta é a espécie comumente encontrada na arborização das ruas tanto da região do complexo industrial como na da região urbana de Cubatão, possibilitando a comparação de re sultados.

A concentração de fluoreto encontrada nas folhas das arvores chapéu do sol, localizadas nas quatro áreas urbanas de Cubatão (Figura 1), sugere que a poluição por fluoreto neste local não deve ser considerada grave porque 0 valor de até $20 \mu \mathrm{g} \mathrm{F} / \mathrm{g}$ de peso seco de folha tem sido considerado normal ${ }^{8}$.

Entretanto, a concentração encontrada nas folhas das árvores localizadas na região das fá- bricas de fertilizantes é preocupante porque valores maiores que $90 \mu \mathrm{g} \mathrm{F} / \mathrm{g}$ podem causar impacto ambiental sever $0^{9}$. A alta concentração encontrada, tanto em 1996 como em 2006 (Figura 1), é coerente com aquela encontrada em Cubatão em $1994^{5}$ e $20011^{10}$ na vegetação do vale do rio M ogi. Por outro lado, esta concentração encontrada ao redor das fábricas de fertilizantes parece não atingir a zona urbana de Cubatão, onde baixa concentração nas folhas foi encontrada nas regiões avaliadas (Figura 1). Assim, o impacto no ecossistema deve atingir não só a fauna eflora ao redor das fábricas de fertilizantes, como pode afetar a saúde de pessoas trabalhand ${ }^{11}$ ou vivendo nas proximidades dessas industrias.

Deveser destacado quea poluição por fluoreto em Cubatão tem sido atribuída à emissão de poluentes pelasfábricas defertilizantes ${ }^{2} e$, de fato, não encontramos alta concentração de fluoreto nas fol has de chapéu do sol localizadas ao redor das demais indústrias locais (dados não mostrados). Também deve ser enfatizado queo poluente deve ser gasoso ${ }^{1}$ e não particulado aéreo, desde quea concentração encontrada éa mesma, lavando-se ou não as folhas (dados não mostrados).

Em conclusão, os dados sugerem que poluição ambiental por fluoreto em Cubatão não melhorou nos últimos dez anos o quedeve servir de alerta para os possíveis efeitos ambientais.

\section{Colaboradores}

PT Tagawa foi responsável pela coleta das amostras em 2006, colaboração nas análises e participação na redação do artigo. DL M oruzzi foi responsável pela coleta das amostras em 1996, redação da monografia de 1996 e participação na redação do artigo. JA Cury é o autor intelectual do trabalho, responsável pelas análises, interpretação dos dados e redação do artigo.

\section{Agradecimentos}

Ao técnico do laboratório de Bioquímica Oral da FOP-UNICAM P, Sr. Waldomiro Vieira Filho, pela colaboração nas análises de íon flúor, e à cirurgiã-dentista Claudia Rodrigues pelo auxílio durante a coleta de amostras feita em 1996. 


\section{Referências}

1. Smith FA, Ekstrand J. The occurrence and chemistry of fluoride. In: Fejerskov O, Ekstrand J, Burt BA, editors. Fluoride in Dentistry. $2^{\text {nd }}$ ed. Copenhagen: M unksgaard; 1996. Chapter 1.

2. Klumpp A, Domingos M, Klumpp G. Assessment of the vegetation risk by fluoride emissions from fertilizer industries at Cubatão, Brazil. Sci Total Environ 1996;192:219-228.

3. Hoflich BL, Weinbruch $S$, Theissmann R, Gorzawski $H$, Ebert $M$, Ortner $H M$, Skogstad A, Ellingsen DG, Drablos PA, Thomassen Y. Characterization of individual aerosol particles in workroom air of aluminum smelter potrooms. J Environ M onit. 2005; 7(5):419-424.

4. Mukherjee AK, Ravichandran B, Bhattacharya SK, Ahmed S, Roy SK, Thakur S, Roychowdhury A, Saiyed HN. Environmental pollution in rural areas of Orissa state due to industrial emissions- with special reference to fluoride. Indian J Environ $\mathrm{H}$ ealth. 2003; 5(4):325-334.

5. Klumpp A, Domingos M, M oraes RM, Klumpp G. Effects of complex air pollution on tree species of the Atlantic rain forest near Cubatão, Brazil. Chemosphere 1998; 36:989-994.

6. Moruzzi DL, Sewel MCD, Cury JA. Avaliação de indicadores de poluição ambiental por fluoretos à partir do Pólo Industrial de Cubatão, SP [monografia]. Araras (SP): Faculdade de Odontologia, Universidade de Araras; 1996.

7. Ayres M, Ayres Jr M, Ayres DL, Santos AS. BioEstat 2.0: Aplicações Estatísticas em Ciências Biológicas e Medicina. Belém: Sociedade Civil M amirauá; Brasília: CN Pq; 2000.

8. Weinstein LH. Fluoride and plant life. J Occup M ed 1977;19:49-78.

9. Scholl G. Ein biologisches Verfahren zur Bestimmung der Herkunft und Vorbreitung von Fluorverbibdungen in der Luft. Landw Forsch 1971;26:29-35.

10. Furlan CM, Domingos M, Salatino A. Effects of initial climatic conditions on growth and accumulation of fluoride and nitrogen in leaves of two tropical tree species exposed to industrial air pollution. Sci Total Environ 2007; 374(2-3):399-407.

11. Seki CT, Buschinelli JT, Ferreira LL, Matallo MR, M orita SM. Comunicação sobre fluorose. Rev bras saúde ocup 1982; 9:48-49.

Artigo apresentado em 18/06/2007

Aprovado em 14/12/2007 